\title{
Gestational age-dependent relationship between cerebral oxygen extraction and blood pressure
}

\author{
Zachary A. Vesoulis ${ }^{1}$, Steve M. Liao ${ }^{1}$ and Amit M. Mathur ${ }^{1}$
}

BACKGROUND: Premature infants may lack mature cerebrovascular autoregulatory function and fail to adapt oxygen extraction to decreasing systemic perfusion.

METHODS: Infants $\leq 28$ weeks of gestational age (GA) were recruited. Systemic oxygen saturation $\left(\mathrm{SpO}_{2}\right)$, mean arterial blood pressure (MABP), and cerebral saturation (near-infrared spectroscopy, $\mathrm{SctO}_{2}$ ) were measured continuously over the first $72 \mathrm{~h}$. Resulting data underwent error-processing. For each remaining $10 \mathrm{~m}$ window, the mean MABP and fractional tissue oxygen extraction (FTOE) were calculated. The infants were divided into two groups (23-25 and 26-28 weeks). The median FTOE at low, medium, and high MABP values (empirically defined within each group based on the 25th and 75th centile) were compared between estimated gestational age (EGA) groups.

RESULTS: Sample $n=68$, mean \pm SD GA $=25.5 \pm 1.3$ weeks, and birthweight $(B W)=823 \pm 195 \mathrm{~g}$. The median FTOE in the more preterm group vs. more mature group was statistically different at lower value of MABP $(P<0.01)$ and higher values of MABP $(P=0.01)$, but not at medium values $(P=0.55)$.

CONCLUSION: The more mature group (GA 26-28 weeks) displayed an appropriate increase in oxygen extraction during hypotension, steadily decreasing as MABP increased, suggesting mature autoregulation. An opposite response was noted in the more preterm group, suggesting an inability to mount a compensatory response when BP is outside of the physiologic range.

$I^{n}$ order to maintain adequate substrate for cerebral metabolism, there is a tightly regulated relationship between oxygen extraction (OE), cerebral blood flow (CBF), and tissue hemoglobin (1). Impairment in cerebrovascular autoregulation in the setting of hypotension $(2,3)$ has been linked to the development of intraventricular hemorrhage (IVH), a potentially devastating form of brain injury in preterm infants, associated with a significantly increased risk for neurodevelopmental impairment (4-6).

However, despite preliminary data suggesting that hypotension and impaired autoregulation are correlated (7) and that rates of brain injury are higher for infants who spend greater proportions of time with a mean arterial blood pressure (MABP) below $30 \mathrm{~mm} \mathrm{Hg}$ (8), the relationship remains unclear. Further complicating matters are the known association between volume resuscitation $(9,10)$ and inotropic medications $(11,12)$ and the development of IVH, generating significant concern for brain injury at both extremes of blood pressure. Indeed, it is the alternating cycles of hypoxicischemia and reperfusion that may injure the germinal matrix and set the stage for pathologic hemorrhage (12).

Recent work by the authors suggests that there is a developmentally linked component of autoregulatory function-less mature infants demonstrate a more limited ability to adapt fluctuations in systemic blood pressure (13). Given that there is also a steadily increasing risk in the development of intraventricular hemorrhage, $\sim 3 \%$ per week of gestation $(14,15)$, it is reasonable to hypothesize that an immature, poorly functioning cerebrovascular autoregulatory system is a significant contributing factor to the development of IVH. However, as noted by Soul et al. (7), impaired autoregulation is common in preterm infants, even in those who do not go on to develop IVH. Taking all of these findings into account, we hypothesize that impaired autoregulation and fluctuations in blood pressure are necessary for the development of IVH.

The traditional depiction of the cerebrovascular autoregulatory system is a sigmoidal curve, with stable CBF across a range of normal blood pressures, decreased CBF during hypotension, and increased CBF during hypertension. $\mathrm{CBF}$ is regulated by changes in vascular tone, constantly changing the vessel caliber to maintain adequate cerebral perfusion. A number of vascular control mechanisms have been described, including the myogenic response, marked by constriction or relaxation of the vessels in response to volume-related stretching of the vessels (16) and autonomic regulation (17), utilizing the release of vasoactive compounds (such as epinephrine) in response to stimulation of the sympathetic or parasympathetic nervous system. Although both of these components of autoregulation are important, there is increasing recognition that cerebral metabolism also has an important role in the regulation of blood flow (18). The cerebral metabolic rate of oxygen $\left(\mathrm{CMRO}_{2}\right)$ is the product of $\mathrm{OE}, \mathrm{CBF}$, and total hemoglobin concentration ( $\mathrm{HbT}$ ),

${ }^{1}$ Division of Newborn Medicine, Edward Mallinckrodt Department of Pediatrics, Washington University School of Medicine, St Louis, MO. Correspondence: Zachary A. Vesoulis (vesoulis_z@kids.wustl.edu)

Received 29 March 2017; accepted 5 August 2017; advance online publication 13 September 2017. doi:10.1038/pr.2017.196 
$\mathrm{CMRO}_{2}=\mathrm{OE} \times \mathrm{CBF} \times[\mathrm{HbT}]$ (ref. 1). Assuming a globally stable $\mathrm{CMRO}_{2}$ and $[\mathrm{HbT}]$ (at least over the short term), an intact autoregulatory system, seeking to maintain stable cerebral tissue oxygenation, should increase extraction when blood pressure is low (i.e., inadequate oxygen delivery) and decrease extraction when the blood pressure rises.

In this paper, we seek to examine the interaction between variation in blood pressure, changes in $\mathrm{OE}$, and developmental maturity. We utilized high-resolution measurement of the arterial blood pressure, pulse oximetry, and cerebral oxygenation (as derived by near-infrared spectroscopy) to evaluate differences in this relationship between a more preterm group (23-25 weeks) and a more mature group (26-28 weeks).

\section{METHODS}

\section{Cohort Selection}

Premature infants, born before 28 completed week of gestation and admitted to the neonatal intensive care unit at St Louis Children's Hospital, a level IV unit serving an urban, suburban, and rural population, were recruited within the first $24 \mathrm{~h}$ for a prospective, multimodality monitoring study. The infants were excluded if: (i) there was an antenatal diagnosis of congenital or chromosomal anomaly; (ii) if an umbilical arterial catheter was not placed; or (iii) the infant was moribund and not expected to survive through the 72 $\mathrm{h}$ monitoring window. The infants were divided by gestational age at birth into a more preterm group (23-25 weeks) and a more mature group (26-28 weeks). The study protocol and procedures were reviewed and approved by the Human Subjects Research Protection Office at Washington University.

\section{Sample Characteristics}

Comprehensive sample characteristics were collected for all included infants. Antenatal factors included mode of delivery, antenatal magnesium sulfate exposure, antenatal corticosteroid exposure, diagnosis of preeclampsia, chorioamnionitis (histopathologic diagnosis), arterial cord blood gas $\mathrm{pH}$, and the 5-min Apgar score. Patient factors included estimated gestational age (EGA) in completed weeks, birth weight, intrauterine growth restriction status (defined as birth weight $<10$ th centile), sex, and race/ethnicity. Clinical factors included CRIB-II score (using the algorithm developed by Parry et al. (19)), IVH (based on cranial ultrasound in the first week of life, graded using the Papile scoring system (20)), inotrope use in the first $72 \mathrm{~h}$ of life (inclusive of dopamine, dobutamine, epinephrine, and norepinephrine), culture-positive sepsis, and mortality.

\section{Institutional Guidelines for the Management of Hypotension} For blood pressure support, our institutional practice is to consider inotrope initiation if: (i) MABP $<$ EGA in weeks, (ii) poor urine output $(<1 \mathrm{ml} / \mathrm{kg} / \mathrm{h})$, and/or (iii) clinical signs of poor perfusion (e.g., prolonged central capillary refill time $>3 \mathrm{~s}$ or tachycardia). Dopamine is used as the first-line agent, with a starting dose between 2.5 and $5 \mathrm{mcg} / \mathrm{kg} / \mathrm{min}$. Epinephrine is used as an alternative first-line treatment (starting dose $0.05 \mathrm{mcg} / \mathrm{kg} / \mathrm{min}$ ) or as a second-line treatment when there is inadequate response to dopamine (when dosing exceeds $10 \mathrm{mcg} / \mathrm{kg} / \mathrm{min}$ ). In addition, hydrocortisone, dosed at $1 \mathrm{mg} / \mathrm{kg}$ every $8 \mathrm{~h}$, can be added as an alternative second-line treatment for refractory hypotension. Volume resuscitation with normal saline (NS) solution or packed red blood cells (pRBCs) can be given before or in conjunction with inotropic support and are administered in $10 \mathrm{ml} / \mathrm{kg}$ (NS) or $15 \mathrm{ml} / \mathrm{kg}$ (pRBC) aliquots.

\section{Data Acquisition}

All data were collected with a sampling rate of $1 \mathrm{~Hz}$ using a data acquisition program (CAS Medical Systems, Branford, CT) that integrated the NIRS, pulse oximetry, and blood pressure signals in a single, time-synchronized file on a laptop computer.

NIRS. Cerebral tissue oxygen saturation $\left(\mathrm{SctO}_{2}\right)$ was obtained using four-wavelength $(690,780,805$, and $850 \mathrm{~nm})$ near-infrared spectroscopy (FORE-SIGHT, CAS Medical Systems, Branford, CT) with a transducer containing a laser diode emitter and one optical detector located $25 \mathrm{~mm}$ from the light source. The non-adhesive optode was placed on the frontoparietal scalp, and recording was conducted over the first $72 \mathrm{~h}$ after birth. The recordings were briefly interrupted every $12 \mathrm{~h}$ to reposition the sensor medially or laterally to prevent skin bruising or breakdown.

Pulse oximetry. Pulse oximetry data (systemic oxygen saturation $\left(\mathrm{SpO}_{2}\right)$ ) were obtained using the Nellcor OxiMax algorithm integrated into the bedside patient monitor (Philips MP70 equipped with multimeasurement module M3001A-A04, Philips Healthcare, Andover, MA) using an adhesive probe placed on the hand or foot (Neonatal-Adult $\mathrm{SpO}_{2}$ Sensor, Covidien, Mansfield, MA).

Blood pressure. Per clinical practice, invasive umbilical blood pressure measurements are made using a pressure transducer (TruWave, Edwards Lifesciences, Irvine, CA), which interfaces the umbilical arterial catheter (3.5 Fr Argyle single-lumen umbilical vessel catheter, Medtronic, Minneapolis, MN) with the patient monitor (IntelliVue MP70, Philips Medical, Andover, MA). Umbilical arterial catheters are placed in the "high-lying" position in the thoracic aorta, located between the sixth and eight vertebral body on chest radiograph. The MABP was recorded at $1 \mathrm{~Hz}$ by taking the time-integrated mean of the beat-to-beat arterial blood pressure.

\section{Data Analysis}

Pre-processing and error correction. Before calculation of fractional tissue oxygen extraction (FTOE)-MABP pairs, the data underwent error correction using a modification of an algorithm developed previously (13). The SctO 2 and $\mathrm{SpO}_{2}$ data streams were extracted from the source data file. Both data streams underwent multistep preprocessing to eliminate missing or invalid data. The data were partitioned into 10 -min epochs (600 serial, nonoverlapping samples) and inspected for (i) interrupted regions of the recording (as noted in the research record), (ii) regions of the recording tagged by the NIRS or $\mathrm{SpO}_{2}$ device where it was not able to properly measure saturations (e.g., probe not in contact with the skin), and (iii) regions with sudden, non-physiologic changes in the baseline or excessive variance, based on the sliding-window motion artifact rejection algorithm proposed by Ayaz et al. (21). The entire data epoch was rejected if either data stream failed one or more of these checks or if continuous measurements were not available for both data sources throughout the entire epoch.

FTOE by blood pressure calculation. In order to examine the interaction between variation in blood pressure and developmentally linked function of the cerebrovascular autoregulatory system, the fractional tissue oxygen (FTOE) was calculated for each 1-s sample for all remaining error-corrected 10-min epochs of data using the standard equation: FTOE $=\left(\mathrm{SpO}_{2}-\mathrm{SctO}_{2}\right) / \mathrm{SpO}_{2}$ (ref. 22). Values of FTOE were then sorted by the corresponding mean MABP in increments of $1 \mathrm{~mm} \mathrm{Hg}$, calculated during the same $10-\mathrm{min}$ epoch, and stored in a matrix. After calculations for all included infants were complete, the mean FTOE, at each measured value of MABP, was then calculated by gestational age group. 


\section{Articles | vesoulis et al.}

Statistical approach. Univariate comparisons of the sample characteristics were made between the more preterm group (2325 weeks) and the more mature group (26-28 weeks) using the Mann-Whitney $U$-test for continuous variables or Fisher's exact test (two-sided) for categorical variables. The median FTOE was compared between three blood pressure states: low, medium, and high. Normative data suggest that most preterm infants have a MABP between 30 and $40 \mathrm{~mm} \mathrm{Hg}$ during the first $72 \mathrm{~h}$ of life $(23,24)$. In addition, a number of studies support $30 \mathrm{~mm} \mathrm{Hg}$ as the lower limit of autoregulatory control $(8,25-27)$, and $40-45 \mathrm{~mm} \mathrm{Hg}$ as the upper limit $(28,29)$. Using the existing literature as a guidepost, the blood pressure groups were defined empirically based on the interquartile distribution of blood pressures within each gestational age group. Comparison was made using Wilcoxon rank sum test. Given the novel nature of the analytic method, a priori sample size calculation was not performed, rather this was a convenience sample recruited over the 3-year study period. Statistical comparisons were made using $\mathrm{R}$ version 3.3.2 (The $\mathrm{R}$ Foundation for Statistical Computing, Vienna, Austria).

\section{RESULTS}

\section{Sample Characteristics}

A total of 68 infants were recruited with a mean \pm SD EGA of $25.4 \pm 1.3$ weeks and mean \pm SD birth weight of $827.1 \pm 193.7$ g. In general, the two EGA groups (23-25 and
26-28 weeks) were roughly equal in size ( $n=36$ vs. $n=32$ ) and were comparable across most clinical characteristics, with the expected exceptions of the mean EGA (24.4 vs. 26.6 weeks, $P<0.01$ ), mean birth weight (742.5 vs. $922.3 \mathrm{~g}, P<0.01)$, and median CRIB-II score (13 vs. $10, P<0.01)$. A complete listing of the characteristics by groups is shown in Table 1 .

\section{Hemodynamic Characteristics}

A total of 1.1 million MABP values were captured with a sample median MABP of $35 \mathrm{~mm} \mathrm{Hg}$ and interquartile range of 31-40 mm Hg. The median blood pressure value in the less mature group was slightly lower than the more mature group ( 32 vs. $35 \mathrm{~mm} \mathrm{Hg}$ ) and had lower bounds of distribution with an interquartile range of $29-36$ vs. $32-39 \mathrm{~mm} \mathrm{Hg}$, which closely match a priori expectations. The distribution of blood pressures between groups is shown in Figure 1. There was no statistically significant difference in inotrope use between the two groups $(33 \%$ vs. $22 \%, P=0.42)$. Inotropic support was started at a median blood pressure of $23 \mathrm{~mm} \mathrm{Hg}$, with a range between 16 and $28 \mathrm{~mm} \mathrm{Hg}$.

Table 1. Sample characteristics

\begin{tabular}{|c|c|c|c|}
\hline & More preterm (23-25 weeks), $N=36$ & More mature (26-28 weeks), $N=32$ & $P$ value \\
\hline EGA, mean (SD), weeks & $24.4(0.6)$ & $26.6(0.8)$ & $<0.01^{*}$ \\
\hline Birth weight, mean (SD), g & $743(133)$ & $922(208)$ & $<0.01^{*}$ \\
\hline Male sex, $n(\%)$ & $25(69)$ & $21(66)$ & 0.79 \\
\hline Complete course, $n(\%)$ & $20(56)$ & $13(41)$ & 0.24 \\
\hline Antenatal magnesium sulfate, $n(\%)$ & $12(33)$ & $18(56)$ & 0.09 \\
\hline Preeclampsia, $n(\%)$ & $2(6)$ & $3(9)$ & 0.66 \\
\hline CRIB-II score, median (range) & $13(10-16)$ & $10(6-14)$ & $<0.01^{*}$ \\
\hline Inotrope use in first $72 \mathrm{~h}, n$ (\%) & $12(33)$ & $7(22)$ & 0.42 \\
\hline Median MABP in first $72 \mathrm{~h}$, (IQR) & $32(29-36)$ & $35(32-39)$ & $0.01^{*}$ \\
\hline Fentanyl use in first $72 \mathrm{~h}, n(\%)$ & $13(36)$ & $19(59)$ & 0.09 \\
\hline $\mathrm{pCO}_{2}$ in first $72 \mathrm{~h}$, mean (SD), $\mathrm{mm} \mathrm{Hg}$ & $49.9(11.2)$ & $43.7(8.4)$ & $0.01^{*}$ \\
\hline \multicolumn{4}{|l|}{$\mathrm{IVH}$} \\
\hline Any grade, $n(\%)$ & $14(39)$ & $9(28)$ & 0.44 \\
\hline Grade III/IV, $n$ (\%) & $4(11)$ & $2(6)$ & 0.67 \\
\hline
\end{tabular}

EGA, estimated gestational age; IQR, interquartile range; IVH, intraventricular hemorrhage; MABP, mean arterial blood pressure. 


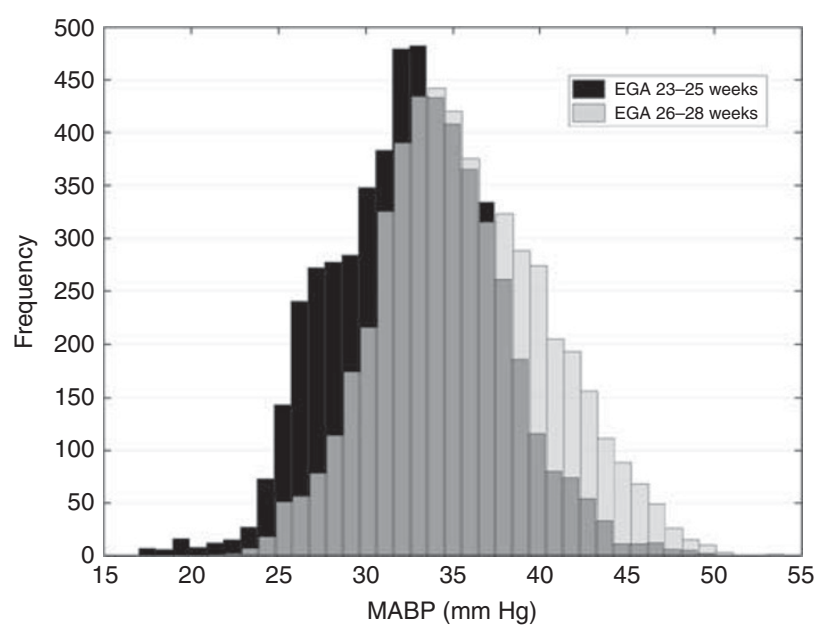

Figure 1. Histogram of MABP values recorded in the two groups. The less mature group is shown with black bars and the more mature group with white bars. Overlap between groups is shown in gray. MABP, mean arterial blood pressure.

\section{Data Quality}

Data collection was started at a mean \pm SD postnatal age of $17.8 \pm 9.7 \mathrm{~h}$ with a mean \pm SD length of $40.2 \pm 13.5 \mathrm{~h}$. A total of $48.5 \%$ of data epochs were rejected owing to one or more error conditions. Approximately 39\% of rejections were due to erroneous/missing blood pressure values, $59 \%$ of rejections due to erroneous/missing NIRS measurements, and $2 \%$ due to motion artifact. Data rejection occurred at equivalent rates between the two groups, with $50.9 \%$ of rejections occurring in the more preterm group and $49.1 \%$ in the more mature group. None of the infants had missing data due to removal of arterial catheter before the end of the study period.

\section{FTOE and Blood Pressure}

Using the empirically defined values, the low, medium, and high MABP thresholds were $<29,29-36$, and $>36 \mathrm{~mm} \mathrm{Hg}$ for the more preterm group and <32, 32-39, and $>39 \mathrm{~mm} \mathrm{Hg}$ for the more mature group. The median FTOE in the more preterm group vs. more mature group was statistically different in the low $(0.218$ vs. $0.267, P<0.01)$ and the high $(0.270$ vs. $0.241, P=0.01)$ MABP range, but not in the medium range $(0.243$ vs. $0.250, P=0.55)$. This differential response is shown graphically in Figure 2, with a steady increase in FTOE as MABP decreases in the more mature group, whereas the more preterm group had a paradoxical decrease in FTOE with decreasing MABP. Although small sample sizes contribute to wide error bars, this pattern is also apparent when EGA is treated as a continuous variable (Figure 3).

\section{DISCUSSION}

These results suggest a developmentally linked, metabolically driven differential response in cerebral OE to low and high blood pressures, which may reflect impaired autoregulation. The more mature group (gestational age (GA) 26-28 weeks)

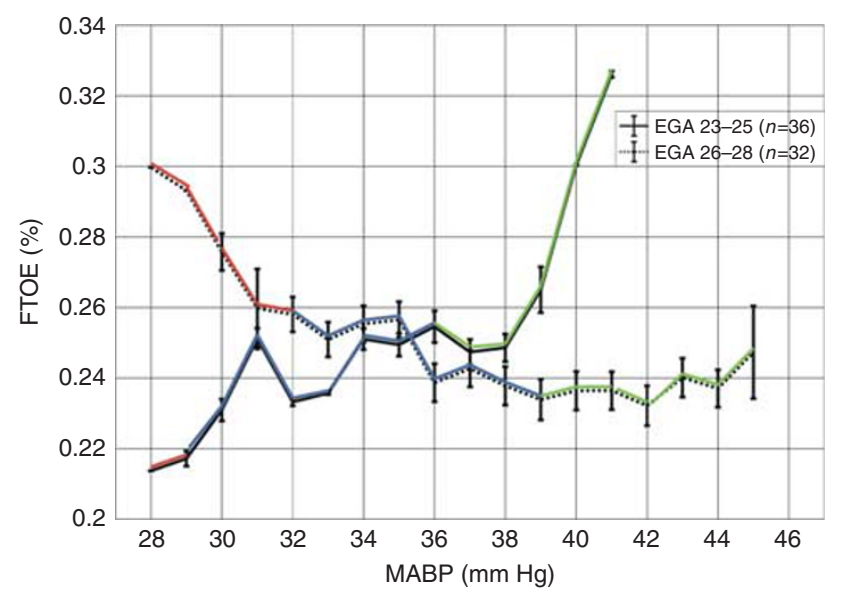

Figure 2. Blood pressure vs. FTOE is shown for both groups (solid line, 23-25 weeks, dashed line, 26-28 weeks). Blood pressure group is defined by color with low MABP in red, medium in blue, and high in green. Note the increase in FTOE during hypotension for the more mature infants, suggestive of a metabolically driven autoregulatory response. In contrast, the more preterm group has a paradoxical drop in FTOE, suggesting immaturity of metabolically driven autoregulation. Whiskers represent \pm 1 SEM. FTOE, fractional tissue oxygen extraction; $M A B P$, mean arterial blood pressure.

displayed an appropriate increase in OE when the blood pressure dropped below $30 \mathrm{~mm} \mathrm{Hg}$, a plateau across a range of medium blood pressures, and a gradual decrease as the MABP exceeds $40 \mathrm{~mm} \mathrm{Hg}$. This matches the expectation that metabolically driven autoregulation will cause OE to increase during periods of decreased CBF (hypotension) and decrease during periods of increased CBF (hypertension) in order to maintain adequate oxygen delivery for cellular function. An opposite response was noted in the more preterm group (GA 23-26 weeks), with a paradoxical decrease in OE, despite decreased CBF (hypotension), potentially an indicator of cellular injury due to ischemia.

The difference in $\mathrm{OE}$ between the two groups is limited to the extremes of blood pressure; both groups maintained FTOE values within the 80th centile values for gestational age (22) when the blood pressure was between 30 and $40 \mathrm{~mm} \mathrm{Hg}$. This finding suggests that the mechanisms that govern blood flow are unable to mount a compensatory response outside the range of medium blood pressures in the most preterm of infants.

Although the data from this study do not provide an answer as to the underlying mechanism that explains this differential response, other literature may offer some insights. During periods of low blood pressure, when cerebral tissue becomes ischemic, vasoactive compounds (including $\mathrm{H}^{+}, \mathrm{K}^{+}, \mathrm{O}^{+}, \mathrm{NO}$, and adenosine) are released from the tissue, triggering local vasodilation $(16,18,30)$. As cellular ionic gradients are not fully established in early development (12), it is reasonable to speculate that this may contribute to a diminished response as compared with the more mature infants. Similarly, vascular control at high blood pressures is largely regulated through 


\section{Articles | Vesoulis et al.}

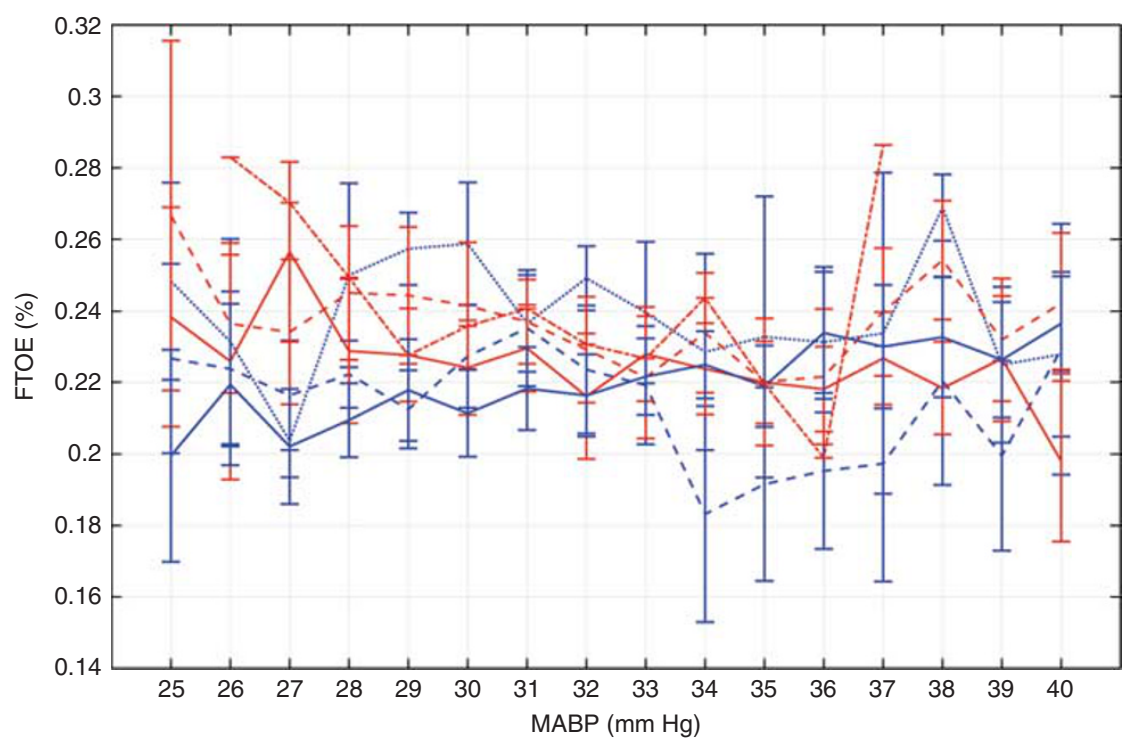

Figure 3. Blood pressure vs. FTOE by individual EGA groups. The more premature group is shown in blue (solid blue $=23$ weeks, dashed blue $=24$ weeks, and dotted blue $=25$ weeks) and the more mature group shown in red (solid red=26 weeks, dashed red = 27 weeks, and dotted red $=28$ weeks). Whiskers represent \pm 1 SEM. EGA, estimated gestational age; FTOE, fractional tissue oxygen extraction.

the myogenic response (16), a factor that has a known association with maturation (31).

As previously noted, hypoxic-ischemic injury to the germinal matrix is one potential mechanism behind the genesis of intraventricular hemorrhage. This study highlights the apparent inability of the preterm brain to compensate accordingly to hypotension, generating the circumstances in which IVH might result. This finding shows potential implications for clinical management, suggesting that aggressive treatment of hypotension may not be indicated in more mature infants with a more functional autoregulatory system. In contrast, conservative management of blood pressure in the most premature of infants carries the real risk of exacerbating the brain injury from ischemia and a more proactive management strategy might be indicated to push the blood pressure higher, and thus CBF back into the range of values that support adequate oxygen delivery for cerebral metabolism.

This study has several important caveats. Primarily, the small sample size precludes broad generalization of the findings in a larger population. Study of this phenomenon in a larger sample is needed to confirm the results and to study the developmental effects on a more granular level. Second, the approach to error correction in this study was intentionally conservative, excluding data frames with potentially problematic areas. Future studies should investigate the tolerance of algorithms for data containing a greater degree of noise. Third, as the pulse oximetry probe location is rotated every $12 \mathrm{~h}$, in order to protect skin integrity in the fragile preterm infant, the pulse oximetry data represent a composite of both pre- and post-ductal saturation, which may have slightly altered the FTOE values. Fourth, the observational study design did not allow for strict control of clinical parameters. Fifth, not surprisingly, there were small differences between the cohort (beyond the intentional EGA grouping) particularly $\mathrm{pCO}_{2}$, a factor that is an important contributor to autoregulatory function. However, the difference between groups ( 49.9 vs. $43.7 \mathrm{~mm} \mathrm{Hg})$, although statistically significant, is not clinically significant, as both values are within the acceptable range for a preterm infant. Sixth, although the blood pressure thresholds were empirically derived, it is possible, and indeed likely, that these thresholds are variable by week of gestation at birth. Study of this phenomenon in a larger sample size will permit the use of distinct BP thresholds by gestational age and further exploration of gestational age as a continuous variable. Finally, although the findings of this present study suggest that the autoregulatory systems of very preterm infants lack the ability to compensate for hypotension by increasing OE, this state only implies an increased risk for injury, not the absolute certainty of it. Extensive clinical validation should be performed to better ascertain the predictive value of this metric.

In summary, using continuous multimodality recording, the metabolically driven response of the preterm brain to low, medium, and high blood pressure values can be measured. More mature infants, those between 26 and 28 weeks, demonstrate the expected response, increasing extraction at low blood pressure values, and decreasing extraction at high values. More preterm infants, those between 23 and 25 weeks, demonstrated the opposite response, providing further evidence that autoregulatory immaturity is a potential risk factor for brain injury by depriving the cerebral tissue of oxygen during the greatest period of demand. 


\section{STATEMENT OF FINANCIAL SUPPORT}

This work was supported by the following grants: Washington University Institute of Clinical and Translational Sciences KL2 Training Program (NIH/ NCATS KL2 TR000450) and The Barnes-Jewish Hospital Foundation and the Washington University Institute of Clinical and Translational Sciences Clinical and Translational Funding Program (NIH/NCATS UL1 TR000448).

Disclosure: The authors declare no conflict of interest.

\section{REFERENCES}

1. Chong SP, Merkle CW, Leahy C, Srinivasan VJ. Cerebral metabolic rate of oxygen (CMRO_2) assessed by combined Doppler and spectroscopic OCT. Biomed Opt Express 2015;6:3941.

2. St. Peter D, Gandy C, Hoffman SB.. Hypotension and adverse outcomes in prematurity: comparing definitions. Neonatology 2017;111:228-33.

3. Miall-Allen VM, de Vries LS, Dubowitz LM, Whitelaw AG. Blood pressure fluctuation and intraventricular hemorrhage in the preterm infant of less than 31 weeks' gestation. Pediatrics 1989;83:657-1.

4. Wilson-Costello D, Friedman H, Minich N, Fanaroff AA, Hack M. Improved survival rates with increased neurodevelopmental disability for extremely low birth weight infants in the 1990s. Pediatrics 2005;115:997-1003.

5. Mukerji A, Shah V, Shah PS. Periventricular/intraventricular hemorrhage and neurodevelopmental outcomes: a meta-analysis. Pediatrics 2015;136: 1132-43.

6. Stoll BJ, Hansen NI, Bell EF, et al. Neonatal outcomes of extremely preterm infants from the NICHD Neonatal Research Network. Pediatrics 2010;126:443-56.

7. Soul JS, Hammer PE, Tsuji M, et al. Fluctuating pressure-passivity is common in the cerebral circulation of sick premature infants. Pediatr Res 2007;61:467-73.

8. Miall-Allen VM, de Vries LS, Whitelaw AG. Mean arterial blood pressure and neonatal cerebral lesions. Arch Dis Child 1987;62:1068-9.

9. Clark CE, Clyman RI, Roth RS, Sniderman SH, Lane B, Ballard RA. Risk factor analysis of intraventricular hemorrhagein low-birth-weight infants. J Pediatr 1981;99:625-8.

10. Goldberg RN, Chung D, Goldman SL, Bancalari E. The association of rapid volume expansion and intraventricular hemorrhage in the preterm infant. J Pediatr 1980;96:1060-3.

11. Köksal N, Baytan B, Bayram Y, Nacarküçük E. Risk factors for intraventricular haemorrhage in very low birth weight infants. Indian J Pediatr 2002;69:561-4.

12. Volpe JJ. Volpe JJ. Neurology of the Newborn. 5th edn, Philadelphia, PA, USA: Saunders/Elsevier, 2008.

13. Vesoulis ZA, Liao SM, Trivedi SB, Ters NE, Mathur AM. A novel method for assessing cerebral autoregulation in preterm infants using transfer function analysis. Pediatr Res 2016;79:453-9.
14. Bajwa N, Berner M, Worley S, Pfister R. Population based age stratified morbidities of premature infants in Switzerland. Swiss Med Wkly 2011;141:w13212.

15. Tioseco JA, Aly H, Essers J, Patel K, El-Mohandes AAE. Male sex and intraventricular hemorrhage. Pediatr Crit Care Med 2006;7:40-4.

16. Cipolla MJ. The Cerebral Circulation. San Rafael, CA: Morgan \& Claypool Life Sciences, 2009.

17. Goadsby PJ. Autonomic nervous system control of the cerebral circulation. In: Robert FS, Karam M, Rami OA, eds. Handbook of Clinical Neurology. Elsevier, Amsterdam, The Netherlands, 2013, pp 193-201.

18. Paulson OB, Strandgaard S, Edvinsson L.. Cerebral autoregulation. Cerebrovasc Brain Metab Rev 1990;2:161-92.

19. Parry G, Tucker J, Tarnow-Mordi W. CRIB II an update of the clinical risk index for babies score. Lancet 2003;361:1789-91.

20. Papile LA, Burstein J, Burstein R, Koffler H. Incidence and evolution of subependymal and intraventricular hemorrhage: a study of infants with birth weights less than 1,500 gm. J Pediatr 1978;92:529-34.

21. Ayaz H, Izzetoglu M, Shewokis PA, Onaral B. Sliding-window motion artifact rejection for functional near-infrared spectroscopy. Conf Proc IEEE Eng Med Biol Soc 2010;2010:6567-70.

22. Alderliesten T, Dix L, Baerts W, et al. Reference values of regional cerebral oxygen saturation during the first 3 days of life in preterm neonates. Pediatr Res 2015;79:55-64.

23. Vesoulis ZA, El Ters NM, Wallendorf M, Mathur AM. Empirical estimation of the normative blood pressure in infants $<28$ weeks gestation using a massive data approach. J Perinatol 2016;36:291-5.

24. Kent AL, Meskell S, Falk MC, Shadbolt B. Normative blood pressure data in non-ventilated premature neonates from 28-36 weeks gestation. Pediatr Nephrol 2009;24:141-6.

25. van de Bor M, Walther FJ. Cerebral blood flow velocity regulation in preterm infants. Biol Neonate 1991;59:329-5.

26. Greisen G. Autoregulation of cerebral blood flow in newborn babies. Early Hum Dev 2005;81:423-8.

27. Munro MJ, Walker AM, Barfield CP. Hypotensive extremely low birth weight infants have reduced cerebral blood flow. Pediatrics 2004;114:1591-6.

28. Ramaekers VT, Casaer P, Daniels H, Marchal G. Upper limits of brain blood flow autoregulation in stable infants of various conceptional age. Early Hum Dev 1990;24:249-58.

29. Tyszczuk L, Meek J, Elwell C, Wyatt JS. Cerebral blood flow is independent of mean arterial blood pressure in preterm infants undergoing intensive care. Pediatrics 1998;102:337-41.

30. Vavilala MS, Soriano SG. Anesthesia for neurosurgery. In: Peter JD, Franklyn PC, Etsuro KM, eds. Smith's Anesthesia for Infants and Children. Elsevier, Amsterdam, The Netherland, 2011:713-44.

31. Mandalà M, Pedatella AL, Morales Palomares S, Cipolla MJ, Osol G. Maturation is associated with changes in rat cerebral artery structure, biomechanical properties and tone. Acta Physiol 2012;205:363-71. 